\title{
Tutorial Interactivo de Trigonometría, como alternativa didáctica para la comprensión teórica y práctica de las matemáticas
}

\author{
Ángel Monzalvo Hernández ${ }^{\mathrm{a}, \mathrm{c}}$, Germán Reséndiz López, ${ }^{\mathrm{a}, \mathrm{d}}$, Heriberto Niccolas \\ Morales $^{\text {b,e }}$, Jaime Garnica González ${ }^{\text {b,f }}$, Noel Iván Toto Arellano ${ }^{\text {a,g }}$ \\ ${ }^{a}$ Universidad Tecnológica de Tulancingo \\ ${ }^{\mathrm{b}}$ Universidad Autónoma del Estado de Hidalgo

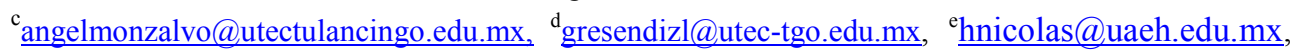 \\ figarnica@uaeh.edu.mx,${ }^{\mathrm{g}}$ noel.toto@utec-tgo.edu.mx
}

\begin{abstract}
Mathematics is an essential cognitive component at any level and educational system and is essential for individuals to achieve good intellectual development. They help to be logical, to reason in an orderly manner, to promote critical thinking and abstraction and to develop models.
\end{abstract}

The aim of this paper is to describe the development and functionality of an interactive tutorial to improve learning of mathematics, specifically in trigonometry topics. The tutorial, considered as a didactic support material, aims to help high school and undergraduate students to understand and learn the trigonometry topics treated in classroom. Although a teacher cannot be 100\% substituted and education can not be completely automated, information and communication technologies (ICT) have been incorporated gradually into educational process allowing students to perform many of their learnings in a self-taught manner.

The approach used to develop the tutorial was the modular teaching model. Development tools (software) used were Flash and Director. Using this type of software allows to generate a product that captures the attention of the student in an effective way, due to the fact that multimedia elements such as: graphics, sounds, texts, animations and videos can be integrated. The Interactive Trigonometry Tutorial (TIT) is composed of five sections: Theory, Solved Problems, Proposed Problems, Knowledge Evaluation and Glossary.

As a result of the development and implementation of TIT, it was possible to train mathematics teachers, prepare high school students competing in mathematics Olympics and prepare personnel who work in companies to present high-level accreditation exams with good results. 
Tutorial Interactivo de Trigonometría, como alternativa didáctica para la comprensión teórica y práctica de las matemáticas

Keywords: Trigonometry, Math learning, Tutorials, Multimedia, Systems Development Life Cycle.

\section{Resumen}

Las matemáticas son un componente cognitivo esencial en cualquier nivel y sistema educativo y resultan fundamentales para que los individuos logren un buen desarrollo intelectual. Ayudan a ser lógicos, a razonar de manera ordenada, a promover el pensamiento crítico y la abstracción y a la elaboración de modelos.

El objetivo del presente trabajo se centra en describir el desarrollo y funcionalidad de un tutorial interactivo para mejorar el aprendizaje de las matemáticas, específicamente en la rama de la trigonometría. El tutorial, considerado como un material de apoyo didáctico, tiene como objetivo ayudar a estudiantes de nivel medio superior y superior a la comprensión y aprendizaje de los temas de trigonometría tratados en el aula. Aunque a un docente no se le puede sustituir al 100\% y la educación no se puede automatizar completamente, las tecnologías de la información y la comunicación (TIC) han logrado incorporarse gradualmente en el proceso educativo permitiendo que los estudiantes puedan realizar muchos de sus aprendizajes de manera autodidacta.

El enfoque utilizado para desarrollar el tutorial fue el modelo modular de enseñanza. Las herramientas de desarrollo (software) utilizadas fueron Flash y Director. Utilizar este tipo de software permite generar un producto que capta la atención del estudiante de manera efectiva, debido a que se pueden integrar elementos multimedia como lo son: gráficos, sonidos, textos, animaciones y videos. El Tutorial Interactivo de Trigonometría (TIT), está integrado por cinco secciones: Teoría, Problemas resueltos, Problemas propuestos, Evaluación de conocimientos y Glosario.

Como resultado del desarrollo y puesta en operación del TIT se logró capacitar a profesores de matemáticas, preparar a estudiantes de nivel medio superior que compiten en olimpiadas de matemáticas y preparar a personal que labora en empresas para presentar exámenes de acreditación del nivel medio superior con buenos resultados.

Palabras clave: Trigonometría, Aprendizaje de matemáticas, Tutoriales, Multimedia, Ciclo de vida de desarrollo de los sistemas. 


\section{Introducción}

En los últimos tres lustros México ha padecido una crisis educativa que se visualiza de manera importante en altos niveles de reprobación, repetición y deserción escolar causadas por la escasa motivación-estímulo del alumno, planes de estudio rígidos y un entorno socioeconómico que provee un clima negativo para un buen aprendizaje en general y de las matemáticas en lo particular. Lo anterior favorece la desmotivación en el estudiante y esto a su vez genera el sentimiento de inferioridad de que las matemáticas son las materias más difíciles y que sólo son comprendidas y usadas por los alumnos más capaces e inteligentes (Juárez y Limón, 2013). En el año 2015, se dieron a conocer los resultados correspondientes a la aplicación de la prueba PLANEA (Plan Nacional para la Evaluación de los Aprendizajes) realizada por el Instituto Nacional para la Evaluación de la Educación (INEE) y la Secretaría de Educación Pública (SEP) de México, que mostraban que el $81 \%$ de los estudiantes que finalizaron su educación media superior tuvieron 'deficientes' habilidades matemáticas, dado que, $51 \%$ de los jóvenes se ubicaban en el nivel I (bajo o insuficiente) y $29.9 \%$ estaban en el nivel II (básico), lo que confirmaba lo indicado por otras pruebas internacionales (León, 2015). Para el año 2017, los resultados de la prueba PLANEA arrojan que el $66.2 \%$ de los estudiantes se ubicaron en nivel I y el $23.3 \%$ en el nivel II, lo que suma un $89.5 \%, 8$ puntos más que en 2015 , lo que significa que los estudiantes no han consolidado los aprendizajes clave para realizar inferencias a partir de un modelo matemático (Instituto Nacional para la Evaluación de la Educación, 2017). Los datos anteriores ponen de manifiesto que en México la calidad de la educación media no es satisfactoria, principalmente en materias de matemáticas donde es necesario el uso del pensamiento lógico y formal por parte de los estudiantes, situación que también han mostrado diversos estudios en el campo de la investigación en educación matemática en México, que señalan que en todos los niveles educativos se reportaba escasa comprensión de los conceptos matemáticos por parte de los estudiantes (Secretaría de Educación Pública, 2002; Ávila, 2016).

Duval (2004) plantea que el análisis de los problemas en el aprendizaje de la trigonometría y de los obstáculos a los cuales se enfrentan regularmente los estudiantes, conduce a que se reconozca una ley fundamental del funcionamiento cognitivo del pensamiento, en este caso del pensamiento proporcional, basado en la coordinación de los sistemas semióticos por parte del sujeto. Las representaciones semióticas hacen referencia a todas aquellas construcciones de sistemas de expresión y representación que pueden incluir diferentes sistemas de escritura, como números, notaciones simbólicas, representaciones tridimensionales, gráficas, redes, diagramas, esquemas, entre otras y cumplen funciones de comunicación, expresión, objetivación y tratamiento (Tamayo, 2006). El reconocimiento 
Tutorial Interactivo de Trigonometría, como alternativa didáctica para la comprensión teórica y práctica de las matemáticas

del objeto matemático debe darse en los sistemas semióticos diferentes: la escritura algebraica de una relación y su representación geométrica, el enunciado de una fórmula en lenguaje cotidiano y la escritura de una fórmula en forma literal, y de manera significativa (Duval, 2004; Tamayo, 2006). Por otro lado, Juarez y Limón (2013), han señalado tres aspectos importantes en relación al tema de la enseñanza de las matemáticas: a) El aprendizaje de las matemáticas debe ser un proceso creativo y explicativo de la realidad, b) Los docentes están en la obligación de adecuar ad hoc la metodología empleada hasta el momento para impartir las asignaturas de matemáticas, y c) A nivel de la educación superior se debe investigar acerca de técnicas de motivación en matemáticas que puedan ser enseñadas a los profesores de todos los niveles para ser aplicadas en las aulas.

Por lo anterior, se considera oportuno y pertinente la elaboración de materiales didácticos para el aprendizaje de las matemáticas que favorezcan la motivación de los estudiantes por apropiarse del conocimiento en esa área. El Tutorial Interactivo de Trigonometría que se presenta en este trabajo es producto del trabajo colaborativo entre profesores invetigadores de la Universidad Tecnológica de Tulancingo (UTT) y de la Universidad Autónoma del Estado de Hidalgo (UAEH) y ha sido utilizado por alumnos de la carrera de Técnico Superior Universitario en Ingeniería Industrial y del área electromecánica que ofrece la UTT.

\section{Los tutoriales digitales como apoyo a la docencia}

En México se han realizado esfuerzos por parte de los académicos que enseñan matemáticas por implementar acciones orientadas al uso de las tecnologías de la información y la comunicación (TIC's) en la enseñanza. Se considera que los materiales multimedia representan una alternativa viable para atender las necesidades de aprendizaje de los jóvenes que cursan la educación media superior y la superior, ya que brindan una mayor participación, estandarizan contenidos, promueven el autoaprendizaje y tienen efectos positivos sobre la motivación y actitudes del alumnado (Carmona, 2002; Rojano, 2003; Santillán, 2006).

Una software tutorial es una herramienta que los alumnos pueden utilizar como un apoyo didáctico o un sistema de aprendizaje (Pontes, 2005). Por lo general un tutorial cuenta con varios módulos. El primero de ellos contiene los contenidos educativos, donde el alumno puede acceder a información teórica o conceptual a la hora de realizar las actividades de aprendizaje. En un segundo módulo o fase se realizan actividades tales como ejercicios o aplicaciones de los contenidos explicados en la fase anterior y por último un módulo de retroalimentación. Un sistema tutorial debe generar la motivación e interés en los temas, además de centrar la atención del alumno para lograr su aprendizaje (Pontes, 2005). 
La multimedia es el conjunto de medios de comunicación o informativos que sirve para presentar información mediante la computadora, empleando textos, gráficos, animaciones, videos y sonidos, que aprovecha precisamente la integración e interacción de muchos medios que utiliza más de un canal de comunicación donde lo interesante en la la interactividad que existe entre el usuario y el software utilizado (Stemler, 1997; Aloraini, 2012). Mediante estos sistemas se obliga al usuario a intervenir en el proceso de transferencia de información, participando activamente en el mismo (Colmenar, 1999). La multimedia forma parte de lo que se conoce como Tecnología Educativa y permite utilizar varios de los sentidos del ser humano para el aprendizaje y la asimilación de la información y los objetivos de aprendizaje se comprenden de una mejor manera (Stemler, 1997). La interactividad es la respuesta que existe del alumno hacia el software y viceversa, por ejemplo si el alumno selecciona alguna sección el software realizara alguna acción como mostrar imágenes o reproducir sonidos.

\section{Metodología de desarrollo del Tutorial Interactivo de Trigonometría}

Para la realización del Tutorial Interactivo de Trigonometría se utilizó el enfoque sistemático de análisis y diseño de sistemas de información, conocido como metodología del ciclo de vida de desarrollo de los sistemas (SDLC por sus siglas en inglés). Systems Development Life Cycle (SDLC) es uno de los modelos más tradicionales para la creación de software. Esta metodología propone siete etapas para la creación de software, que son: Identificación de problemas, Determinación de requerimientos, Análisis de necesidades, Diseño, Desarrollo y documentación, Pruebas y mantenimiento, Implantación y evaluación (Whitten y Bentley, 2008; Kendall y Kendall, 2011; Laudon y Laudon, 2012).

A continuación se describen de manera breve y simplificada los pasos realizados para el desarrollo del tutorial de acuerdo a la metodología de Ciclo de Desarrollo de Sistemas (SDLC).

1. Identificación de problemas: En esta etapa se detectaron los principales problemas que generaban la deserción de alumnos por la no comprensión de temas y sus posibles soluciones. Se detectó que los estudiantes no dedicaban el tiempo necesario para estudiar algún tema en especifico y falta de motivación para estudiar matemáticas.

2. Determinación de requerimientos: Se estudió si era o no conveniente generar un material interactivo para el apoyo de la enseñanza de la trigonometría. Debido a los factores mencionados en la etapa 1 , se considero necesario contar con un apoyo extraescolar y no solo depender del maestro. A partir de entrevistas con alumnos y profesores se determinaron los requerimientos para el desarrollo del proyecto. Se determino que la herramienta de estudio fuera capaz de: a) Consultarse cada que el estudiante lo requiera y 
Tutorial Interactivo de Trigonometría, como alternativa didáctica para la comprensión teórica y práctica de las matemáticas

de forma individual. b) Tener los temas más esenciales que se traten dentro de la asignatura (Trigonometría). c) Contener ejercicios que el alumno pueda resolver para la adquisición de mayores habilidades. d) Evaluar los conocimientos que el alumno a adquirido. e) Sentirse en un ambiente amigable, para poder llamar la atención del alumno.

3. Análisis: Se realizó una revisión de las entradas, procesos y salidas de información que eran necesarias para el tutorial por medio de diagramas de flujo.

4. Diseño: El diseño del material se conceptualizó a partir de los resultados del análisis. El tutorial se diseñó con los contenidos que cubrian la problemática detectada en la etapa de análisis. Además en esta etapa se definieron la formas de interactuar entre el software tutorial y el alumno. El diseño se dividió en dos: Diseño educativo y Diseño de interfaces. El tutorial se constituyo de cinco secciónes: Teoría (cuatro unidades), Problemas resueltos, Problemas propuestos, Evaluación de conocimientos y Glosario.

5. Desarrollo: El desarrollo es la parte donde se transfiere todo lo establecido en el diseño a código de programación, es decir, se programan las actividades que el material interactivo realizara frente al alumno.

6. Pruebas y mantenimiento: Las pruebas son las evaluaciones que se le realizan al material interactivo, para ver si cumple con los objetivos planteados anteriormente, además de verificar que el comportamiento del software sea conforme a lo diseñado y programado. Se realizaron dos pruebas. La primera con profesores de las asignaturas de matemáticas y la segunda con una muestra aleatoria de alumnos de la carrera de Tecnico Superior Universitario en Ingeniería Industrial y de Electromecánica.

7. Implantación y evaluación: Si las evaluaciones realizadas en la etapa anterior fueron superadas en esta etapa se procede a utilizar el software. Para el caso del Tutorial Interactivo de Trigonometría se realizó la instalación del software en las salas de cómputo de la UTT para que todo aquel alumno que deseara hacer uso del mismo lo pudiera utilizar para reforzar sus conocimientos o como material de apoyo para estudiar los temas de trigonometría que incluye.

\subsection{Herramientas de desarrollo del software tutorial}

Las herramientas de desarrollo y el entorno bajo el cual se desarrolló el tutorial fueron Director y Flash, debido a que permiten integrar de una forma eficiente los gráficos, imágenes, sonidos y textos y han mostrado versatilidad y efectividad para la elaboración de presentaciones multimedia, libros electrónicos, CD's interactivos, paginas Web, entre otras aplicaciones (Quito, 2017). Director es una herramienta robusta para la creación de diversos proyectos multimedia, gracias a su capacidad para soportar diversos formatos de imágenes, gráficos, textos, sonidos, los cuales pueden ser integrados para la creación de una sola 
presentación o proyecto. Flash cuenta con herramientas que permiten crear y publicar presentaciones, y para generar gráficos con movimientos y aplicaciones que utilizan datos. Al igual que Director con Flash se pueden enriquecer las aplicaciones creadas integrando sonido, animaciones, entre otros recursos. Para la parte de aplicaciones realizadas por medio de datos, Flash cuenta con ActionScript, un lenguaje de programación para la manipulación de actividades a realizar dentro de las interfaces o publicaciones de los documentos Flash.

Flash Player es el software encargado de ejecutar los archivos swf creados por Flash, además gracias a Flash Player las aplicaciones se pueden visualizar de la misma manera sin importar la plataforma o navegador que se utilice. ActionScript 2.0 fue el lenguaje de programación que se utilizó en la creación de las acciones para las interfaces del tutorial. Este lenguaje permite utilizar Flash para crear sitios interactivos, aplicaciones multimedia, demostraciones de productos, materiales de capacitación, y más. Un Script es una serie de instrucciones que le indican a un programa cómo ejecutar o desarrollar un procedimiento.

\section{Descripción del Tutorial Interactivo de Trigonometría}

En la interfaz de Menú principal del tutorial se muestran las secciones que lo integran y por medio de un clic sobre el nombre de la sección a la que se desea acceder se enlaza a la sección correspondiente, como se muestra en la Figura 1 (a). Al realizar un enlace con alguna de las unidades que se encuentran dentro de la sección de Teoría se muestra la interfaz ilustrada en la Figura 1 (b), en la cual se pueden seleccionar temas que contiene la unidad (en este caso fue seleccionada la Unidad 3). Al seleccionar algún tema del menú de las unidades se visualiza una pantalla como la mostrada en la Figura 1 (c), donde se presentan dos recuadros; el que se sitúa a la derecha contendrá teoría del tema seleccionado y el recuadro ubicado a la izquierda mostrara una imagen o imágenes referentes al tema. En la Figura 1 (d) se muestra la interfaz que pertenece al menú de Problemas resueltos. Mediante este menú se puede elegir algún ejercicio resuelto, solo basta presionar alguno de los botones que aparecen en la interfaz para que se muestre el ejercicio. La interfaz muestra uno de los ejercicios de la sección Problemas Resueltos. La Figura 1 (e) muestra un ejemplo de interfaz donde se aprecia el ejercicio, para visualizar los pasos de la solución se utilizan los botones circulares que se encuentran en la parte inferior derecha hasta llegar a la respuesta de cada ejercicio. Al igual que en el menú de Problemas Resueltos, en la interfaz perteneciente a Problemas Propuestos se podrá elegir algún ejercicio para resolver. En la Figura 1 (f) se muestra la interfaz con un ejemplo de los ejercicios contenidos en la sección de Problemas Propuestos. En esta interfaz se puede apreciar el ejercicio y las opciones que se proponen para la solución del ejercicio. Para elegir una opción solo es necesario presionar el botón izquierdo del mouse sobre la opción deseada y posteriormente se 
Tutorial Interactivo de Trigonometría, como alternativa didáctica para la comprensión teórica y práctica de las matemáticas

visualiza un botón para la verificación de respuesta. El tutorial tiene una interfaz para la sección de Evaluación de Conocimientos. Para mostrar los ejercicios a resolver se deberá presionar el botón con la leyenda "Siguiente" que aparece en la parte inferior derecha de la pantalla. La Figura 1 (g) muestra la interfaz de uno de los ejercicios que contiene la evaluación así como las posibles opciones de respuesta del problema. Después de elegir una opción se podrá evaluar si la elección fue correcta o no mediante el botón con la leyenda "Respuesta" mostrándose un mensaje correspondiente a la elección al lado derecho del botón de evaluación.

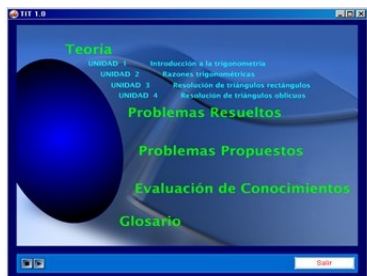

(a)

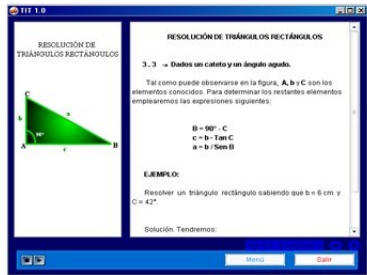

(c)

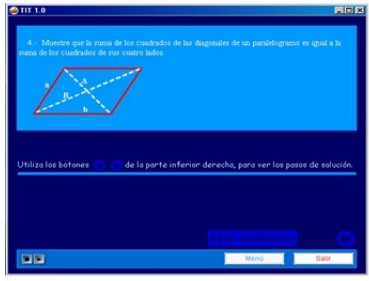

(e)

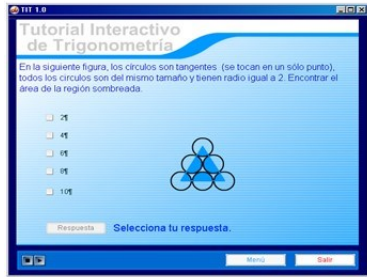

(g)
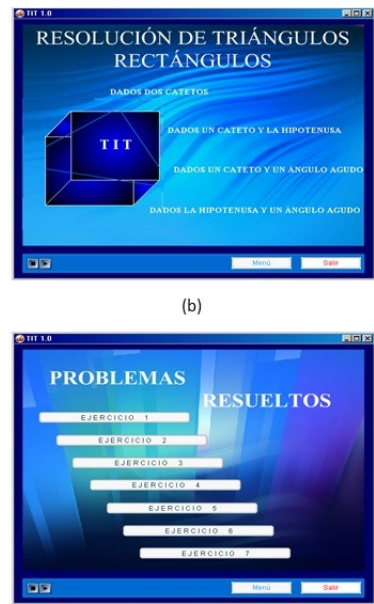

(d)

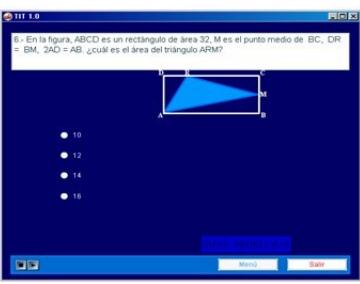

(f)

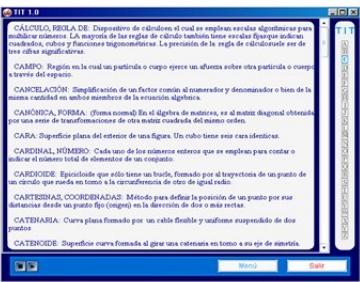

(h)

Fig. 1 Intefaces de los módulos del Tutorial Interactivo de Trigonometría 
Por último la Figura 1 (h) muestra la interfaz que aparece al seleccionar la sección Glosario desde el menú principal, en donde se podrán consultar conceptos relacionados a la trigonometría.

\section{Resultados y consideraciones finales}

El desarrollo del Tutorial Interactivo de Trigonometría presentado en este trabajo es una aplicación multimedia para la enseñanza de la trigonometría a nivel medio superior y superior que proporciona a los estudiantes la oportunidad de estudiar y aprender a partir de situaciones de aprendizaje interactivo por medio de una computadora. Ha tenido impacto dentro de la comunidad escolar, debido a que los alumnos realizan consultas, practicas y evaluaciones de una forma diferente a las que se realizan en el aula y le proporciona información de una forma diferente al alumno. El tutorial tiene la ventaja de ser portado y utilizado con facilidad. Gracias a la creación del tutorial tanto alumnos como catedráticos cuentan con un apoyo más para el proceso de enseñanza - aprendizaje. Actualmente contar con este tipo de apoyos didácticos es muy importante dentro de las instituciones educativas ya que el ambiente computacional esta impactando en el desarrollo de competencias y capacidades. Se considera de gran valor que la propia institución pueda desarrollar este tipo de materiales a partir del trabajo y creatividad de profesores y alumnos. El diseño del tutorial consideró la utilización del mismo de manera sistemática por parte de los estudiantes para facilitarles su aprendizaje de manera autodidácta, con apoyo de diversos recursos visuales y textuales. Como resultado del desarrollo y puesta en operación del TIT se logró capacitar a profesores de matemáticas, preparar a estudiantes de nivel medio superior que compiten en olimpiadas de matemáticas y preparar a personal que labora en empresas para presentar exámenes de acreditación del nivel medio superior con buenos resultados. partir de la utlización del tutorial por parte de los profesores en los cursos que imparten. Finalmente el tutorial ha propiciado en los estudiantes la observación, el pensamiento analítico y la clarificación de conceptos, lo que ha incidido en la mejora de su logro académico. 
Tutorial Interactivo de Trigonometría, como alternativa didáctica para la comprensión teórica y práctica de las matemáticas

\section{Referencias}

Aloraini, S. (2012). The impact of using multimedia on students' academic achievement in the College of Education at King Saud University. Journal of King Saud University - Languages and Translation. 24, 75-82.

Ávila, A. (2016). La investigación en educación matemática en México: una mirada a 40 años de trabajo. Educación matemática, 28(3), 31-60. Recuperado el 16 de junio de 2018, de http://www.scielo.org.mx/scielo.php?script=sci_arttext\&pid=S1665$58262016000300031 \& \operatorname{lng}=$ es\&tlng=es.

Carmona, J. (2002). Elaboración de tutoriales multimedia para el aprendizaje de conceptos estadísticos. XXI, Revista de educación. 4, 291-298. Recuperado el 25 de junio de 2018, de http://rabida.uhu.es/dspace/bitstream/handle/10272/1918/b15150835.pdf

Colmenar, A. (1999). Propuesta de Diseño Curricular en un Marco Constructivista para los Diferentes Niveles del Nuevo Sistema Educativo: Aplicación a las Energías Renovables. Tesis Doctoral. UNED.

Duval, R. (2004). Semiosis y Pensamiento Humano. Registros Semióticos y Aprendizajes Intelectuales. Colombia: Universidad del Valle.

Instituto Nacional para la Evaluación de la Educación. (2017). PLANEA Resultados nacionales 2017. Educación Media Superior. Lenguaje y Comunicación / Matemáticas. Recuperado el 19 de junio de 2018 , de http://planea.sep.gob.mx/content/general/docs/2017/ResultadosNacionalesPlaneaMS2017.PDF

Juárez, B. y Limón, O. (2013). Las matemáticas y el entorno socioeconómico como causa de deserción escolar en el nivel medio superior en México. Multidisciplina. Revista electrónica de la Facultad de Estudios Superiores Acatlán. 15, 72-90. Recuperado el 8 de abril de 2017 de http://revistas.unam.mx/index.php/multidisciplina/article/view/45299.

Kendall, K.E. y Kendall, J. E. (2011). Análisis y diseño de sistemas. Octava edición. México: Pearson Educación.

Laudon K.C. y Laudon J.P. (2012). Sistemas de información gerencial. Decimosegunda edición. México: Pearson Educación.

León, M. (2015, Agosto, 5). Con bajo nivel en matemáticas, el 81\% de alumnos de bachillerato. El Financiero. Recuperado el 14 de marzo de 2017 de http://www.elfinanciero.com.mx/nacional/con-bajo-nivel-en-matematicas-el-81-de-alumnos-debachillerato.html.

Pontes, A. (2005). Aplicaciones de las tecnologías de la información y de la comunición en la educación científica. Primera parte: Funciones y recursos. Revista Eureka sobre Enseñanza y Divulgación de las Ciencias. 2(1), 2-18. 
Quito, A.M. (2017). Diseño y desarrollo en Flash de un juego didáctico para el aprendizaje de herramientas Lean Manufacturing. Tesis de Maestría. Universitat Politècnica de València. España.

Rojano, T. (2003). Incorporación de entornos tecnológicos de aprendizaje a la cultura escolar: Proyecto de innovación educativa en matemáticas y ciencias en escuelas secundarias públicas de México. Revista Iberoamericana de Educación. 33, 135-165.

Santillán, M. (2006). Tecnologías de la información y de la comunicación en la educación. Revista Mexicana de Investigación Educativa. 11(28), 7-10.

Secretaría de Educación Pública. (2002). Problemas y políticas de la educación básica. Programa y materiales de apoyo para el estudio. Licenciatura en Educación Secundaria. 1er semestre. Tercera Edición. México: SEP.

Stemler, L. (1997). Educational Characteristics of Multimedia: A literature review. Journal of Educational Multimedia and hipermedia. 6(3/4), 339-359.

Tamayo, O. E. (2006). Representaciones semióticas y evolución conceptual en la enseñanza de las ciencias y las matemáticas. Revista Educación y Pedagogía. 18(45), 37-49.

Whitten, J.L. y Bentley, L.D. (2008). Análisis de sistemas: diseño y métodos. Séptima edición. México: McGraw-Hill/Interamericana Editores. 